\title{
A practical approach to diagnose and treat rickets
}

\author{
Aditi Jaiman ${ }^{1}$, Lokesh Tiwari ${ }^{2}$, Jatin Prakash ${ }^{3}$, Ashish Jaiman ${ }^{4}$ \\ ${ }^{1}$ SK Nursing Home and Hospital, Tikonia, G.B. Pant Marg, Haldwani, Uttarakhand, India \\ ${ }^{2}$ Department Paediatrics, All India Institute of Medical Sciences, Patna, India \\ ${ }^{3}$ Vardhman Mahavir Medical College \& Safdarjung Hospital, New Delhi, India \\ ${ }^{4}$ Central Institute of Orthopaedics, Vardhman Mahavir Medical College \& Safdarjung Hospital, New
}

Received: 2020-12-05.

Accepted: $2020-12-27$

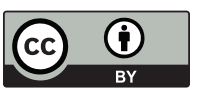

This work is licensed under Creative Commons Attribution 4.0 International License

J Clin Med Kaz 2021; 18(1):7-13

Corresponding author:

Ashish Jaiman.

E-mail: drashishjaiman@gmail.com;

\section{Abstract}

Rickets is a disease of growing bone, before fusion of epiphyses. There is defective mineralization of cartilage matrix in the zone of provisional calcification caused either by nutritional vitamin D deficiency and/or low calcium intake or by non-nutritional causes, like hypophosphatemic rickets and rickets due to renal tubular acidosis. In addition, some varieties are due to inherited defects in vitamin D metabolism and are called vitamin D dependent rickets. The diagnosis is made on the basis of history, physical examination, and biochemical testing, and is confirmed by radiographs. Treatment consists of vitamin D supplementation as Stoss therapy or daily or weekly oral regimens, all with equal efficacy and safety, combined with calcium supplements. For renal rickets, the active form of Vit D, 1,25(OH)2 also called Calcitriol is used, treatment is tailored to another type of renal rickets.

Routine supplementation starting from the newborn period is being increasingly endorsed by various international organizations. Adequate sunlight exposure, food fortification, and routine supplementation are the currently available options for tackling this nutritional deficiency. In this review article, we discuss the pathophysiology, diagnosis, and management of rickets in detail. rickets

Key words: rickets, vitamin D, hypophosphatemic rickets, renal

\section{Introduction}

Osteoid (protein matrix) and hydroxyapatite (mineral phase) forms the basic structure of bone. Hydroxyapatite is mainly composed of calcium and phosphorous. Unmineralized matrix at the growth plates in growing bone is the hallmark of rickets [1]. This form of extreme vitamin D deficiency usually discloses itself between 12-18 months of age. Deficiency state must have been persisting for months before clinical revilement of florid rickets. Rarely, vitamin D deficiency can express itself with growth failure, lethargy, irritability, hypocalcemic seizures, and a predilection to respiratory infections particularly during infancy $[2,3]$. Sufficient intake of vitamin D has a preventive role for rickets [4].

\section{Vitamin D metabolism}

Vitamin D2 (Ergocalciferol), is produced by plants while vitamin D3 (Cholecalciferol) is produced in the skin from 7-dehydrocholesterol on exposure to ultraviolet B (UV-B) light at a wavelength of 290- 320 $\mathrm{nm}$. Vitamin D3 is transported to the liver and further converted to 25-hydroxyvitamin D (25-OH-D) by the action of 25-hydroxylase. 25-OH-D is then converted to 1, 25-dihydroxyvitamin $\mathrm{D}(1,25-(\mathrm{OH}) 2-\mathrm{D})$ in kidneys and in other tissues. 25-OH-D is the nutritional indicator of vitamin D. Vitamin D acts as a pre-hormone. Its active metabolites (25-OH-D and 1, 25(OH) 2) are involved in many metabolic routes that are afar from calcium homeostasis [4-8].

\section{Etiopathogenesis of Rickets}

Alkaline phosphatase, calcitonin, calcitriol and parathyroid hormone are the major enzymes and hormones involved in an intricate manner with many organs, in order to form a proper bone. Any disruption in assembly, absorption or metabolism of Vitamin D has significant influence on calcium metabolism and in development of rickets [9, 10]. Vitamin D maintains serum levels of calcium and phosphorus; therefore, in circumstances of hypocalcemia or hypophosphatemia, vitamin D stimulates bone resorption. Vitamin D deficiency or resistance therefore results in hypocalcemia and hypophosphatemia. 


\begin{tabular}{|l|l|}
\hline \multicolumn{2}{|l|}{ Non - renal rickets } \\
\hline 1. & Nutritional \\
\hline 2. & Gastrointestinal causes: \\
\hline a) & $\begin{array}{l}\text { Malabsorption e.g. Celiac disease b) Hepatobiliary disorders } \\
\text { e.g. Wilson disease, neonatal cholestasis }\end{array}$ \\
\hline & \\
\hline 3. & Medications e.g. anticonvulsant therapy \\
\hline 4. & Oncogenic -Mesenchymal tumors \\
\hline 5. & Rickets of prematurity \\
\hline 6. & Vitamin D dependent rickets type II (VDDR type II) \\
\hline & \\
\hline Renal rickets \\
\hline 1. & Chronic kidney disease. (Renal Osteodystrophy) \\
\hline 2. & $\begin{array}{l}\text { Hereditary hypophosphatemic rickets a) X-linked } \\
\text { hypophosphatemic (XLH) rickets b) Autosomal dominant } \\
\text { hypophosphatemic rickets c) Autosomal recessive } \\
\text { hypophosphatemic rickets d) Hereditary hypophosphatemic } \\
\text { rickets with hypercalciuria. }\end{array}$ \\
\hline 3. & Distal renal tubular acidosis \\
\hline 4. & $\begin{array}{l}\text { Fanconi syndrome a) Primary b) Secondary (Cystinosis, } \\
\text { tyrosinemia, Wilson's disease, Lowe syndrome) }\end{array}$ \\
\hline 5. & Vitamin D dependent rickets type I (VDDR type I) \\
\hline $\begin{array}{l}\text { Dent disease, Tumor induced rickets, McCune Albright syndrome, } \\
\text { Epidermal nevus syndrome, Neurofibromatosis }\end{array}$ \\
\hline
\end{tabular}

Low calcium levels arouses the release of parathyroid hormone (PTH); which, in turn acts through bone and kidney and correct hypocalcemia to some extent. At the same time, it heightens urinary phosphate excretion, leading to hypophosphatemia and rickets [11].

Rickets can be broadly classified according to underlying etiology as non-renal and renal rickets (Table 1). Non-renal causes of rickets include nutritional deficiency, rickets of prematurity, oncogenic osteomalacia and vitamin D dependent rickets type II (VDDR II).

\section{Non-renal rickets}

Vitamin D deficiency rickets (nutritional rickets): It is described by deficiency of vitamin D and calcium. Vitamin D deficiency is the fallout of no or reduced sun exposure with resultant minimal synthesis in skin. It is commonly seen in cold climates, people with restrictive clothing, or in people with extensive burns. Also it can occur in people who eat vitamin D deficient food or if there are issues with intestinal absorption of vitamin $\mathrm{D}$, as in inflammatory bowel disease (IBD) and celiac disease, or in hepatobiliary disorders such as neonatal cholestasis and Wilson diseases. Maternal vitamin D deficiency and prematurity are risk factors for vitamin D deficiency in neonates. Solely breast-milk fed babies particularly till the latter half of infancy, without vitamin D fortification/ supplementation are susceptible for rickets. Certain medicines e.g. anti convulsants and antiretroviral drugs can precipitate vitamin $\mathrm{D}$ deficiency by induction of P-450 enzyme; thereby, enhancing catabolism of $25(\mathrm{OH}) \mathrm{D}$ and $1,25(\mathrm{OH}) 2$. In these patients serum levels of $25(\mathrm{OH}) \mathrm{D}$ are low, with low or normal calcium levels and low phosphorous levels [4, 11].

Rickets of prematurity: Vitamin D levels are low in premature infants, as they didn't get enough time during third trimester to gather vitamin D from the mother. The need of calcification of fetal skeleton results into increased activation of $25(\mathrm{OH}) \mathrm{D}$ to $1,25(\mathrm{OH}) 2 \mathrm{D}$ in the mother's kidneys. Reduced vitamin $\mathrm{D}$ levels in mother during pregnancy thereby lead to fetal vitamin D deficiency, and in stark cases, fetal rickets [11].
Tumor induced osteomalacia (TIO) or oncogenic osteomalacia: It is a condition characterized by severe hypophosphatemia with osteomalacia that has been induced by tumor. It's usually seen in adults. The tumors that have been associated with rickets or osteomalacia are generally benign, small, and coin from the mesenchyma (e.g., sclerosing hemangiopericytoma). Vitamin D metabolism has been found to be abnormal in oncogenic osteomalacia and plasma calcitriol is reduced despite hypophosphatemia [12].

Vitamin D dependent rickets (VDDR) Type 2: It is an autosomal recessive disease with mutation in the vitamin $\mathrm{D}$ receptor (VDR) gene (12q12-q14), causing end-organ resistance to $1,25(\mathrm{OH}) 2$ vitamin $\mathrm{D}$. There are very high levels of 1 , $25(\mathrm{OH}) 2 \mathrm{D}$ with normal or high values of 25-OH-D [13].

Renal rickets: It includes rickets with renal failure i.e. renal osteodystrophy, rickets with acidosis but without renal failure i.e. proximal and distal renal tubular acidosis (RTA) and Fanconi syndrome, rickets without acidosis or renal failure i.e. familial hypophosphatemic rickets and vitamin D dependent rickets (VDDR) Type 1.

Rickets due to renal osteodystrophy (includes rickets with renal failure): It is a type of metabolic bone disease with abnormality of mineralization and its turnover and abnormalities of volume, linear development, and strength. In chronic kidney disease, calcium and phosphate metabolism is altered and is associated with chronic metabolic acidosis and presents with low calcium, low vitamin $\mathrm{D}$, and excess parathyroid hormone (PTH) activity with a high serum phosphate level [11, 13].

Renal rickets with acidosis and without renal failure: Proximal or type 2 RTA is due to decreased reabsorption of filtered bicarbonate in proximal tubule with consequential bicarbonaturia, decrease in serum bicarbonate levels, and subsequent metabolic acidosis. Type I or distal RTA is due to failure to excrete $\mathrm{H}+$ ions from the distal renal tubule, resulting in metabolic acidosis with normal anion gap, hypokalemia, and raised serum chloride, hypercalciuria and hypocitraturia causing nephrolithiasis and nephrocalcinosis [13, 14]. Fanconi syndrome (FS) is a rare proximal tubular malfunction that leads to excess amounts of glucose, uric acid, potassium, sodium, bicarbonate, phosphates, and certain amino acids being excreted in the urine $[13,15]$ Both types of RTA have low vitamin D levels along with low calcium and low phosphorous [13].

Rickets without acidosis or renal failure: It includes hypophosphatemic rickets and VDDR type 1. Hypophosphatemic rickets (previously called vitamin D-resistant rickets) consists of a group of inherited disorder in which the primary problem is the phosphate wasting. They are of two types a) secondary to increased fibroblast growth factor-23 signaling, this can be $\mathrm{X}$ linked, autosomal dominant or autosomal recessive and b) due to a primary renal tubular defect such as hereditary hypophosphatemic rickets with hypercalciuria (HHRH), Dents disease, Toni-Debre-Fanconi and Lowe syndromes.

$\mathrm{X}$-linked hypophosphatemic rickets (XLHR) is an X-linked dominant disorder and is the most common form of genetic rickets and constitutes $80 \%$ of hereditary phosphate wasting disorders. The gene responsible has been identified on chromosome Xp 22.1 and has been named PHEX. Child with XLHR does not develop tetany or myopathy which is seen in hypocalcemic rickets. Growth retardation is marked in untreated males, who seldom reach a height of $110 \mathrm{cms}$. Main biochemical feature of XLHR is the low tubular reabsorption of filtered phosphate (TRP) in the presence of low serum phosphate. Normal range of TRP is $80-95 \%$ and in patients with XLHR it is reduced to 40-70\% [13]. Autosomal dominant hypophosphatemic rickets 
is described by hypophosphatemia, hyperphosphaturia, fatigue, bone pain and lower limb deformities and rickets along with inappropriately low or normal vitamin D3 levels [13].

Autosomal recessive hypophosphatemic rickets (ARHR) display elevated serum levels of FGF-23, renal phosphate wasting and normal levels of $1,25(\mathrm{OH}) 2$ [13]. ARHR is an autosomal recessive form that is written off as reduced renal phosphate reabsorption, hypophosphatemia and rickets. It can be differentiated from other forms of hypophosphatemia by increased serum levels of $1,25(\mathrm{OH}) 2$ vitamin $\mathrm{D}$ resulting in hypercalciuria. Dents disease is characterized by low-molecularweight (LMW) proteinuria, hypercalciuria and at least one of the following: nephrocalcinosis, hematuria, hypophosphatemia or renal insufficiency and rickets. Either type, whether due to FGF23 defect or renal tubule defect have low phosphate, normal calcium, normal 25(OH) D. In type with raised FGF23 signaling, 1, 25(OH) 2 are low while in HHRH, Dents disease 1, 25(OH) levels are high or normal $[11,13,16]$.

Vitamin D dependent rickets (VDDR) Type 1: Mutation in the 1-alpha hydroxylase gene with autosomal recessive inheritance characterizes this disease. It causes impaired 1-alpha hydroxylation of 25-hydroxyvitamin $\mathrm{D}$ in the renal proximal tubule. The defect is located on chromosome 12q13.3. Hypocalcemic symptoms are evident during the first few months of life and the affected children can have oligodentia and enamel hypoplasia. Common symptoms are failure to thrive, tremulousness and convulsions [13, 17-19]. Children may have secondary hyperparathyroidism. The serum levels of $25(\mathrm{OH})$ vitamin D are normal while serum 1, 25(OH) 2 vitamin D levels are low.

Rickets can also be classified according to the major type of mineral deficiency as calcipenic or phosphopenic rickets. Calcipenic rickets is caused by deficiency of calcium and/or Vitamin D. Calcium deficiency is commonly due to insufficient intake or decreased absorption of calcium due to vitamin D deficiency. Serum vitamin D level may be low or normal. Calcipenic rickets is frequently (not always) associated with low serum calcium levels. Phosphopenic rickets is caused by renal phosphate wasting and is characteristically characterized by low serum levels of phosphorus $[11,20]$.

\section{Diagnosis}

Diagnosis is based on clinical features along with radiological changes and distinct biochemical changes.

Clinical Findings: Generally these children have failure to thrive, lethargy, protruding abdomen, proximal muscle weakness, dilated cardiomyopathy and high risk of bony fractures. Specific clinical findings are:

Head: Craniotabes, frontal bossing, delayed closure of fontanel, craniosynostosis with or without signs of increased intracranial pressure, delayed dentition (suggestive if there is no eruption of incisors by age 10 months or molars by age 18 months) and dental caries.

Chest: Physical findings include rachitic rosary and Harrison groove. Frequent respiratory infections are common and one may find atelectasis on the chest-x-ray.

Back: Scoliosis, kyphosis and lordosis may be evident

Appendicular skeleton: bilateral enlargement of wrist and ankles, valgus or varus deformities of the knee, windswept deformity (valgus deformity of one leg with varus deformity of other leg), anterior bowing of tibia and femur, coxa vara and leg pains.

Hypocalcemic symptoms: Children may present with tetany, seizures and at times stridor caused by laryngeal spasm [1].

Radiological findings: Early changes are seen well in the growth plate of ulna in the wrist and fibula in the lower limbs. They are indicated by widening of physis (growth cartilage) and loss of demarcation at the zone of provisional calcification which lies at the epiphyseal-metaphyseal junction. Cupping and splaying of the metaphysis, delayed appearance or reduced size of epiphyseal centers or osteopenia of shaft are other radiological features (Figure 1).

Figure 1 - AP X-ray Of 2 year old girl showing typical features of nutritional rickets with widened physis and cupping and fraying of metaphysis

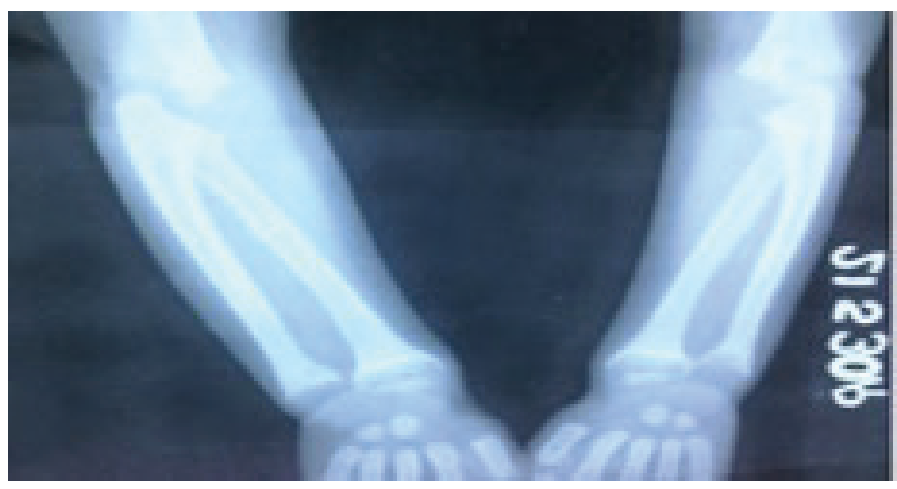

Figure 2 - Clinical photograph of 3 year old child with nutritional rickets with bilateral genu valgum

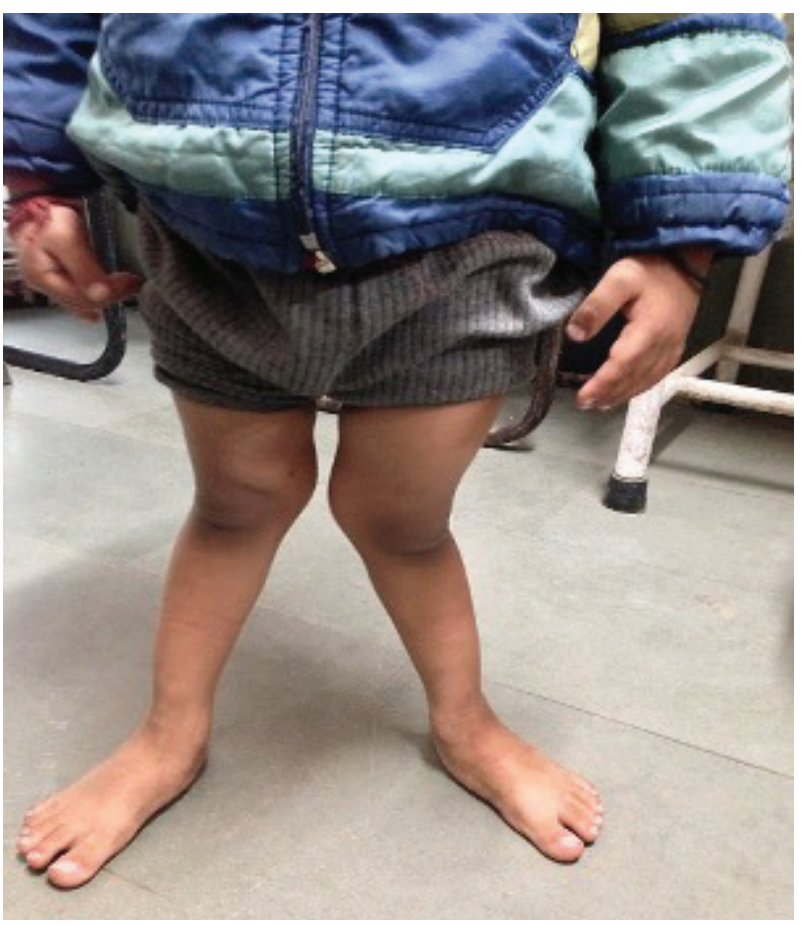

Deformities and pathological features can occur in advanced forms of rickets (Figure 2) [13]. Sabre Tibia that has a pronounced anterior convexity resembling the curve of a sabre is classically described for syphilis may also be present in rickets that gets healed after vitamin D correction (Figure 3a-b).

\section{Biochemical findings:}

a) Serum ALP values: It is a marker of disease activity because it partakes in the mineralization of bone and growth plate cartilage. Both hypocalcemic and hypophosphatemic rickets have raised serum ALP levels [21]. 
Figure 3a - AP X-ray of 4 year old child showing curved tibia Sabre tibia- with signs of rickets

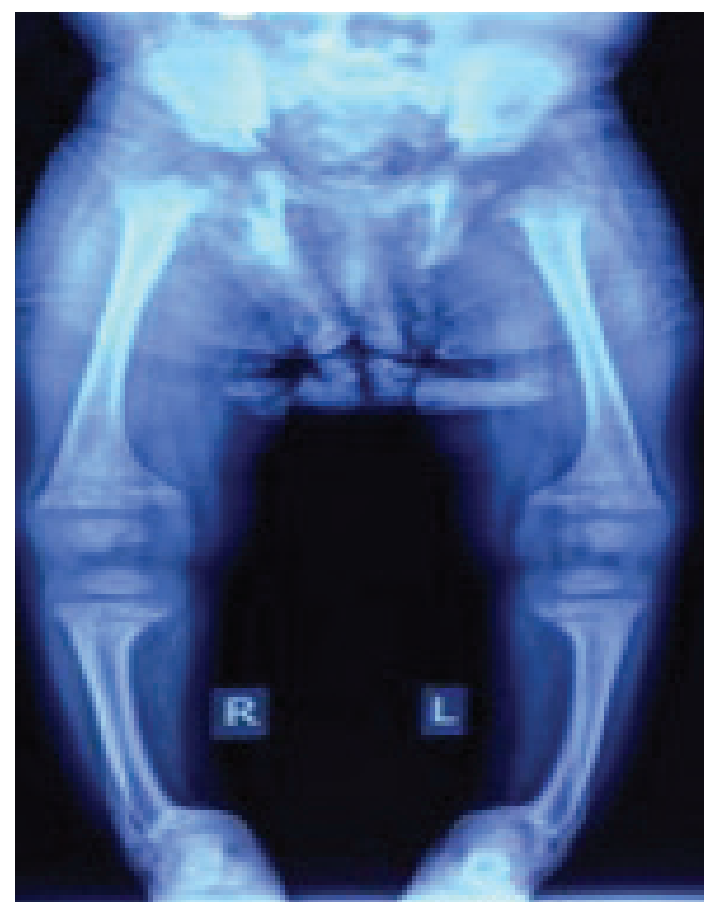

b) Serum calcium values: It is usually low in calcipenic rickets, but may be normal in initial stages of the disease due to increase in PTH levels [11]. It is normal in phosphopenic rickets.

c) Serum phosphorus values: Its values are usually are low in both calcipenic and phosphopenic rickets.

d) Serum PTH values: It is elevated in calcipenic rickets and usually normal in phosphopenic rickets.

The diagnostic flowchart of suspected rickets use values of serum inorganic phosphorus (iP) and PTH to distinguish calcipenic from phosphopenic rickets [22].

e) Serum $25(\mathrm{OH}) \mathrm{D}$ values: It helps to distinguish rickets caused by vitamin $\mathrm{D}$ deficiency from other causes of calcipenic rickets [11]. It reflects the amount of vitamin D stored in the
Figure $\mathbf{3 b}$ - AP X-ray of same child after 6 months of treatment showing resolution of rickets with correcting deformity

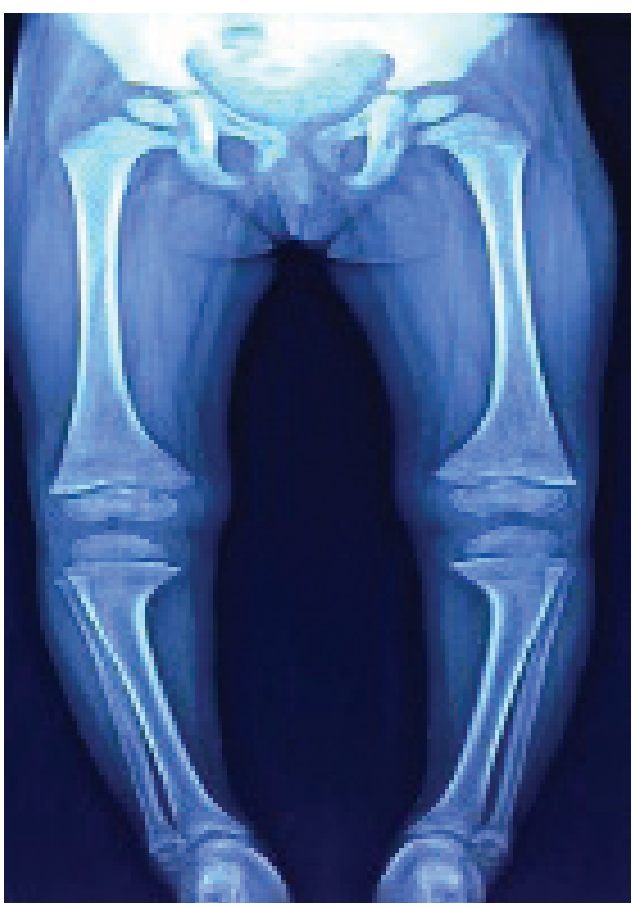

body and thus, is low in vitamin D deficiency, while it is normal or slightly increased in the other forms.

f) Serum 1, 25(OH) 2 vitamin D values: It is the activated form of vit $\mathrm{D}$ and it can be low, normal, or increased in calcipenic rickets. 1, 25(OH) 2 vit D levels initially increase in response to rising levels of $\mathrm{PTH}$, but may afterwards decrease because its base material $25(\mathrm{OH}) \mathrm{D}$ is limited. 1, 25(OH) 2 vit D is increased in VDDR type II and hypophosphatemic rickets.

g) For children with phosphopenic rickets, the causes can be distinguished by measuring urinary amino acids, bicarbonate, glucose, and calcium concentrations. A summary of biochemical changes seen in different types of rickets is discussed in Table 2.

Table 2

Diagnoses of different types of Rickets

\begin{tabular}{|c|c|c|c|c|c|c|c|}
\hline & Calcium & Phosphorus & \begin{tabular}{|l} 
Alkaline \\
Phosphatase
\end{tabular} & $\begin{array}{l}\text { Parathyroid } \\
\text { hormone }\end{array}$ & Vitamin D & Urine phosphorus & Bicarbonate \\
\hline Nutritional & Low & Low & High & High & Low & Low & Normal \\
\hline RTA & Low & Low & High & High & Low & High & $\begin{array}{l}\text { Low urine } \mathrm{pH} \text {, } \\
<5.3 \text { RTA II and } \\
>5.3 \text { type I }\end{array}$ \\
\hline VDDR I & Low & Low & High & High & $\begin{array}{l}\text { High 25(OH)D low } \\
\text { 1,25 (OH)D }\end{array}$ & Low & Normal \\
\hline VDDR II & Low & Low & High & High & $\begin{array}{l}\text { High 25(OH)D and } \\
1,25(\mathrm{OH}) \mathrm{D} \text { both }\end{array}$ & & Normal \\
\hline Hypophosphatemic & Normal & Low & High & Normal & $\begin{array}{l}\text { Normal 25(OH)D } \\
\text { Low 1,25(OH)D }\end{array}$ & High & Normal \\
\hline Renal failure & Low & High & High & High & $\begin{array}{l}\text { Low } 25(\mathrm{OH}) \mathrm{D} \text { and } \\
1,25(\mathrm{OH}) \mathrm{D}\end{array}$ & & Low \\
\hline
\end{tabular}

Levels of $25(\mathrm{OH}) \mathrm{D}$ are assessed for the purpose of defining deficiency [23]. Various methods can be used, the best being TMS (Tandem Mass Spectrometer), but is not performed routinely. Enzyme-linked immunosorbent assay, chemiluminescence or radioimmuno assay are commonly used methods. Although a fasting specimen is recommended, it is not essential; diurnal variations are also not so important [24, 25]. Data suggest that $20 \mathrm{ng} / \mathrm{mL}(50 \mathrm{nmol} / \mathrm{L})$ can be set as the serum 
$25(\mathrm{OH}) \mathrm{D}$ level that concurs with the level that would cover the needs of 97.5 percent of the population [26]; thus, vitamin D concentrations of $>20 \mathrm{ng} / \mathrm{mL}(50 \mathrm{nmol} / \mathrm{L})$ are considered as sufficient, between $12-20 \mathrm{ng} / \mathrm{mL}(30-50 \mathrm{nmol} / \mathrm{L})$ as insufficient and $<12 \mathrm{ng} / \mathrm{mL}(<30 \mathrm{nmol} / \mathrm{L})$ as deficient $[26,27]$.

\section{Treatment}

Depending on cause of rickets, the treatment varies, in terms of which form of vitamin D is used for supplementation along with dosing of calcium and phosphorous.

For nutritional rickets: Contrary to the popular conception; oral administration of vitamin D replenishes vitamin D concentrations more quickly than by the intramuscular (IM) route $[26,27]$. When absorption from the gut or compliance is an issue; intramuscular route is prescribed. Moreover, absorption is independent of fed state [23, 27].

For neonates and infants till 1 year of age, daily $2000 \mathrm{IU}$ of vitamin D with $500 \mathrm{mg}$ of calcium for a 3 -month period is mentioned. At the end of 3 months, response to treatment should be reevaluated and treatment continued $[26,27]$. If larger doses of vitamin D are to be given, then, 60,000 IU of vitamin D weekly for 6 weeks is recommended (only in infants older than 3 months of age). After completion of this therapy with weekly doses, maintenance doses of 400 IU of vitamin D daily and 250$500 \mathrm{mg}$ of calcium are required [27].

From one year onwards till 18 years of age, 3000-6000 IU/ day of vitamin $\mathrm{D}$ along with calcium intake of $600-800 \mathrm{mg} /$ day is recommended for a minimum of 3 months. For larger doses, 60,000 IU of vitamin D weekly for 6 weeks may be given orally $[27,28]$. The maintenance doses of $600 \mathrm{IU} /$ day of vitamin D and 600-800 mg of calcium need to be continued after therapy [27].

An alternative protocol is "Stoss therapy," which consists of a high dose of oral vitamin D (600,000 IU) given on a single day, (age more than 12 months), or 300,000 IU (age less than 12 months) then maintained at 400-1000 IU of vitamin D per day for 8 weeks orally followed by 400 IU/day [11]. The X-ray at 3 weeks in patients responding to therapy shows characteristic white line of healing with complete resolution by 3 months (Figure 4a-c) [1]. Stoss therapy is useful when compliance is a problem. However, Stoss therapy (from the German "push") can result in hypercalcemia and nephrocalcinosis [28]. Doses of 150,000 or $300,000 \mathrm{IU}$ are also similarly effective with lesser side effects [11].

Vitamin D dependent rickets (VDDR) Type 2 (Also known as hereditary resistance to vitamin D - HRVD): Treatment contains unusually high doses of calcitriol and calcium for 3-5 months. Initial dose of $1,25 \mathrm{D}$ is $6 \mathrm{mcg}$ /day along with calcium supplementation. It is gradually increased to very high doses up to $60 \mathrm{mcg} /$ day and calcium up to 3 gram per day with the aim to achieve normal calcium levels and $\mathrm{X}$ ray evidence of healing [13].

Rickets due to renal osteodystrophy: Treatment includes vitamin $\mathrm{D}$ in the form of cholecalciferol, phosphate restriction in diet and use of phosphate binders. Calcium based phosphate binders are safe and effective such as calcium carbonate, calcium acetate and calcium gluconate. In the presence of high serum calcium levels, calcium containing phosphate binders may cause soft tissue calcification. Newer non-calcium-nonaluminum phosphate binding drugs like Sevelamer are used in these situations [13].

Renal rickets with Type I (distal) renal tubular acidosis (dRTA) and without renal failure: Patients with dRTA demands an alkaline dose of 1-3 $\mathrm{mEq} / \mathrm{kg} / \mathrm{day}$, with normalization of
Figure $4 \mathbf{a}$ - AP $X$ ray 2 year old child with classical signs of rickets with cupping of metaphysis

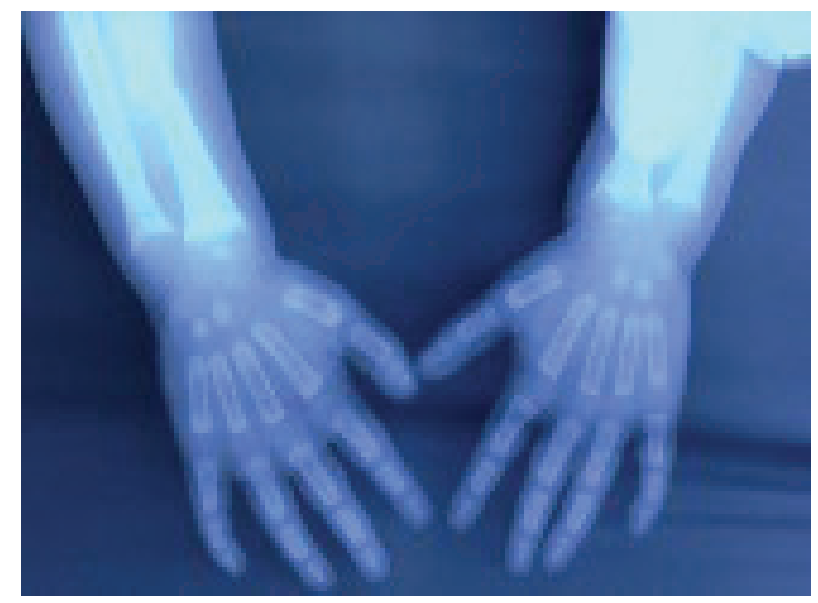

\section{Figure $\mathbf{4 b}$}

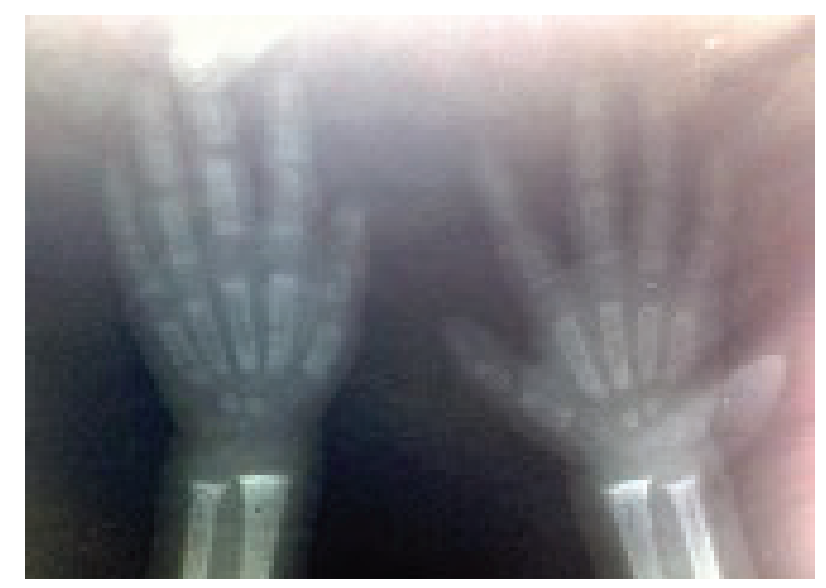

Figure $\mathbf{4 c}$ - AP X-ray of same child at 3 months post Stoss therapy showing resolution of rickets

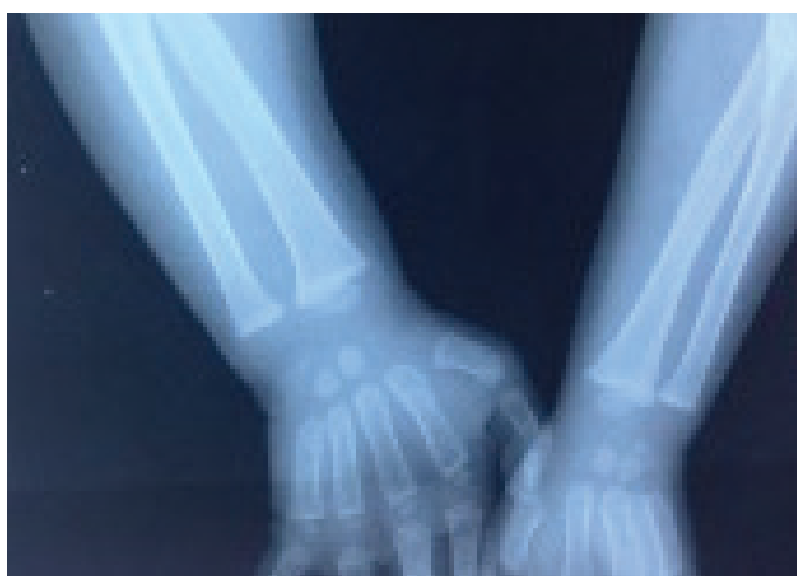

hypercalciuria and hypocitraturia being used for dose titration Symptomatic treatment including replacement of fluids, bicarbonates and potassium, supplement oral phosphates along with vitamin $\mathrm{D}$ as $1,25(\mathrm{OH}) 2$ vitamin $\mathrm{D}$, forms the principles of treatment $[11,13]$.

Renal rickets with hypocalcemia (Secondary hyperparathyroidism) Vitamin D-dependent rickets type 1 (VDDR 1): Treatment is with $1,25(\mathrm{OH}) 2$ vitamin $\mathrm{D}, 0.25-2.0$ mcg per day. This is continued till $\mathrm{X}$ ray evidence of healing is seen. After this, maintenance dose is given as $0.25-1.0 \mathrm{mcg} / \mathrm{day}$ depending on the severity and body weight. This is combined with elemental calcium supplementation of $30-75 \mathrm{mg} / \mathrm{kg} /$ day. During therapy serum calcium is kept at lower limit of normal, normal phosphate and high normal levels of PTH [17, 19]. 
Hypophosphatemic rickets: In rickets due to FGF23, treatment consists of oral administration of phosphate and 1, 25 (OH) 2 vitamin D. The recommended oral phosphate preparation consists of the solution of $136 \mathrm{~g}$ of dibasic sodium phosphate and $58.5 \mathrm{~g}$ phosphoric acid (85\%) in a liter of water. One milliliter of solution contains $30 \mathrm{mg}$ of elemental phosphorus. Prepared oral formulations are available for ready use. The recommended dose of phosphate varies from $30-90 \mathrm{mg} / \mathrm{kg} /$ day, with an average of $60 \mathrm{mg} / \mathrm{kg} /$ day divided into four doses [13]. Treatment with phosphate solution be administered simultaneously with low doses of active vitamin D and one shall gradually increase the dose. The recommended dose is between $0.02-0.03 \mu \mathrm{g} / \mathrm{kg} /$ day [16]. Nephrocalcinosis and hyperparathyroidism must be monitored during the course of therapy. Adjuvant therapy with hydrochlorothiazide is used by some to control hypercalciuria [13].

Whilst in renal tubule defect, as in HHRH (Hereditary hypophosphatemia rickets with hypercalciuria), treatment is phosphate supplementation alone whereas the addition of vitamin D can create complications, such as hypercalcemia, nephrocalcinosis and renal damage [13]. In Dents disease treatment of hypercalciuria is done with dietary sodium restriction and thiazide diuretics. Dietary calcium restriction has been shown to worsen the risk of bone disease. Oral phosphate therapy and active vitamin $\mathrm{D}$ supplementation result in improvement of bone disease. Dialysis and transplantation are the terminal treatment offered to these patients if they develop stage V CKD [13].

\section{Prevention}

Preventive measures in high risk groups as discussed below will lessen the burden of rickets and need for treatment.

Premature neonates: Enteral calcium intake of about 150 to $220 \mathrm{mg} / \mathrm{kg}$ per day, phosphorous intake of $75-140 \mathrm{mg} / \mathrm{kg} / \mathrm{day}$ and vitamin D intake of $400 \mathrm{IU} /$ day is suggested [27, 29].

Neonates and infants up to 1 year of age: 400 IU of vitamin D supplementation is recommended till one year of age. In the first year of life, if dietary calcium intake is not adequate (250$500 \mathrm{mg}$ ), calcium supplementation is recommended [27].
Pregnant mothers and lactating females should receive 600 IU of vitamin D daily [26, 30] along with $1200 \mathrm{mg}$ of calcium [31].

Children older than 1 year and adolescents: 600 IU of vitamin D [26, 27] and 600-800 mg/day calcium [31].

At-risk groups: Children on anti-seizure medications, children on treatment for malignancy, restricted sun exposure such as in children with physical disabilities, children with fat malabsorption, liver disease or renal insufficiency, transplant recipients, those with history of rickets, children with predisposition to osteoporosis such as in hypogonadism or Cushing's syndrome, etc., requires higher doses of vitamin D $[23,26,27]$.

Thus, for at-risk infants, 400-1000 IU/day and from 1 year onwards, 600-1000 IU/day along with adequate calcium intake as per the age group is recommended [27, 32].

\section{Conclusion}

Nutritional Rickets and osteomalacia are avertable diseases that are on the rise in undeveloped world particularly. Screening for vitamin D deficiency is suggested in individuals at risk. Appropriate treatment corrects the upset bone metabolism and deformities. Renal causes for rickets should be considered if there is no or little response to vitamin D supplementation and other associated features. A normal serum creatinine dismisses renal osteodystrophy. The presence of acidosis points towards RTA. Further differentiation between types 1 and type 2 RTA is possible by estimating urine $\mathrm{pH}$. The absence of acidosis indicates either hypophosphatemic rickets or VDDR. Hypophosphatemic rickets shows renal phosphate wasting, while VDDR can be identified by measuring serum vitamin D. Overall, the treatment depends on etiology, hence a detailed systematic assessment is essential

Disclosures: There is no conflict of interest for all authors.

\section{References}

1. Greenbaum LA. Rickets and Hypervitaminosis D. In Robert Kliegman: Editor, Nelsons Textbook of Pediatrics. 1st South Asia edition. Elsevier India. 2016; 331-341.

2. Ladhani S, Srinivasan L, Buchanan C, Allgrove J. Presentation of vitamin D deficiency. Arch Dis Child. 2004; 89(8):781-4. doi: 10.1136/ adc.2003.031385. PMID: 15269083; PMCID: PMC1720051.

3. Najada AS, Habashneh MS, Khader M. The frequency of nutritional rickets among hospitalized infants and its relation to respiratory diseases. J Trop Pediatr. 2004; 50(6):364-8. doi: 10.1093/tropej/50.6.364. PMID: 15537725.

4. Wagner CL, Greer FR; American Academy of Pediatrics Section on Breastfeeding; American Academy of Pediatrics Committee on Nutrition. Prevention of rickets and vitamin D deficiency in infants, children, and adolescents. Pediatrics. 2008; 122(5):1142-52. doi: 10.1542/peds.2008-1862. Erratum in: Pediatrics. 2009; 123(1):197. PMID: 18977996.

5. Webb AR. Who, what, where and when-influences on cutaneous vitamin D synthesis. Prog Biophys Mol Biol. 2006; 92(1):17-25. doi: 10.1016/j.pbiomolbio.2006.02.004. PMID: 16766240.

6. Holick MF. Vitamin D deficiency. N Engl J Med. 2007; 357(3):266-81. doi: 10.1056/NEJMra070553. PMID: 17634462.

7. Misra M, Pacaud D, Petryk A, Collett-Solberg PF, Kappy M; Drug and Therapeutics Committee of the Lawson Wilkins Pediatric Endocrine Society. Vitamin D deficiency in children and its management: review of current knowledge and recommendations. Pediatrics. 2008; 122(2):398-417. doi: 10.1542/peds.2007-1894. PMID: 18676559.

8. Holick MF. Vitamin D: importance in the prevention of cancers, type 1 diabetes, heart disease, and osteoporosis. Am J Clin Nutr. 2004; 79(3):362-71. doi: 10.1093/ajcn/79.3.362. Erratum in: Am J Clin Nutr. 2004; 79(5):890. PMID: 14985208.

9. Nield LS, Mahajan P, Joshi A, Kamat D. Rickets: not a disease of the past. Am Fam Physician. 2006; 74(4):619-26. PMID: 16939184.

10. Drezner MK. Rickets and osteomalacia. In: Goldman L, Ausiello DA, eds. Cecil Textbook of Medicine. 22nd ed. Philadelphia, Pa.: Saunders, 2004:1545.

11. Sahay M, Sahay R. Rickets-vitamin D deficiency and dependency. Indian J Endocrinol Metab. 2012; 16(2):164-76. doi: 10.4103/22308210.93732. PMID: 22470851; PMCID: PMC3313732. 
12. Sahay M, Sahay R. Renal rickets-practical approach. Indian J Endocrinol Metab. 2013; 17(1):S35-44. doi: 10.4103/2230-8210.119503. PMID: 24251212; PMCID: PMC3830358.

13. Akila Devi V , Thangavelu S, Vijayakumar M. Renal Rickets - Pediatrician's Perspective. Indian Journal of Practical Pediatrics 2017; $19(2): 156$

14. Lee JH, Park JH, Ha TS, Han HS. Refractory rickets caused by mild distal renal tubular acidosis. Ann Pediatr Endocrinol Metab. 2013; 18(3):152-5. doi: 10.6065/apem.2013.18.3.152. Epub 2013 Sep 30. PMID: 24904870; PMCID: PMC4027071.

15. Deal JE, Barratt TM, Dillon MJ. Fanconi syndrome, ichthyosis, dysmorphism, jaundice and diarrhoea--a new syndrome. Pediatr Nephrol. 1990; 4(4):308-13. doi: 10.1007/BF00862505. PMID: 2206896.

16. Velásquez-Jones L, Medeiros-Domingo M. Hereditary hypophosphatemic rickets. Bol Med Hosp Infant Mex. 2013; 70:421-430.

17. Fraser D, Kooh SW, Kind HP, Holick MF, Tanaka Y, DeLuca HF. Pathogenesis of hereditary vitamin-D-dependent rickets. An inborn error of vitamin D metabolism involving defective conversion of 25-hydroxyvitamin D to 1 alpha,25-dihydroxyvitamin $D$. $N$ Engl $J$ Med. 1973; 289(16):817-22. doi: 10.1056/NEJM197310182891601. PMID: 4357855.

18. Yan Y, Calikoglu AS, Jain N. Vitamin D-dependent rickets type 1: a rare, but treatable, cause of severe hypotonia in infancy. $J$ Child Neurol. 2011; 26(12):1571-5. doi: 10.1177/0883073811411190. Epub 2011 Jun 23. PMID: 21700898.

19. Kim CJ. Vitamin D dependent rickets type I. Korean J Pediatr. 2011; 54(2):51-4. doi: 10.3345/kjp.2011.54.2.51. Epub 2011 Feb 28. PMID: 21503197; PMCID: PMC3077501.

20. Thacher TD, Fischer PR, Pettifor JM. Vitamin D treatment in calcium-deficiency rickets: a randomised controlled trial. Arch Dis Child. 2014; 99(9):807-11. doi: 10.1136/archdischild-2013-305275. Epub 2014 Apr 19. PMID: 24748637; PMCID: PMC4145444.

21. Whyte MP. Physiological role of alkaline phosphatase explored in hypophosphatasia. Ann N Y Acad Sci. 2010; 1192:190-200. doi: 10.1111/j.1749-6632.2010.05387.x. PMID: 20392236.

22. Baroncelli GI, Bertelloni S, Ceccarelli C, Amato V, Saggese G. Bone turnover in children with vitamin D deficiency rickets before and during treatment. Acta Paediatr. 2000; 89(5):513-8. doi: 10.1080/080352500750027763. PMID: 10852183.

23. Holick MF, Binkley NC, Bischoff-Ferrari HA, Gordon CM, Hanley DA, Heaney RP et al.; Endocrine Society. Evaluation, treatment, and prevention of vitamin D deficiency: an Endocrine Society clinical practice guideline. J Clin Endocrinol Metab. 2011; 96(7):1911-30. doi: 10.1210/jc.2011-0385. Epub 2011 Jun 6. Erratum in: J Clin Endocrinol Metab. 2011 Dec; 96(12):3908. PMID: 21646368.

24. Roth HJ, Schmidt-Gayk H, Weber H, Niederau C. Accuracy and clinical implications of seven 25-hydroxyvitamin D methods compared with liquid chromatography-tandem mass spectrometry as a reference. Ann Clin Biochem. 2008; 45(Pt 2):153-9. doi: 10.1258/ acb.2007.007091. PMID: 18325178.

25. Laboratory Procedure Manual. 2015. Available from http://www.cdc.gov/nchs/data/nhanes/nhanes_05_06/VID_D_met_Vitamin_D. pdf. Accessed November 15, 2020.

26. Munns CF, Shaw N, Kiely M, Specker BL, Thacher TD, Ozono K et al., Global Consensus Recommendations on Prevention and Management of Nutritional Rickets. J Clin Endocrinol Metab. 2016; 101(2):394-415. doi: 10.1210/jc.2015-2175. Epub 2016 Jan 8. PMID: 26745253; PMCID: PMC4880117.

27. From Indian Academy of Pediatrics 'Guideline for Vitamin D and Calcium in Children' Committee., Khadilkar A, Khadilkar V, Chinnappa J, Rathi N, Khadgawat R, Balasubramanian S, Parekh B, Jog P. Prevention and Treatment of Vitamin D and Calcium Deficiency in Children and Adolescents: Indian Academy of Pediatrics (IAP) Guidelines. Indian Pediatr. 2017; 54(7):567-573. doi: 10.1007/s13312-017-1070-x. PMID: 28737142.

28. Hoppe B, Gnehm HE, Wopmann M, Neuhaus T, Willi U, Leumann E. Vitamin D poisoning in infants: a preventable cause of hypercalciuria and nephrocalcinosis. Schweiz Med Wochenschr. 1992; 122(8):257-62. German. PMID: 1311865.

29. Abrams SA; Committee on Nutrition. Calcium and vitamin d requirements of enterally fed preterm infants. Pediatrics. 2013; 131(5):e1676-83. doi: 10.1542/peds.2013-0420. Epub 2013 Apr 29. PMID: 23629620.

30. Elder CJ, Bishop NJ. Rickets. Lancet. 2014; 383(9929):1665-1676. doi: 10.1016/S0140-6736(13)61650-5. Epub 2014 Jan 10. PMID: 24412049.

31. Indian Council of Medical Research (ICMR), Nutrient Requirements and Recommended Dietary Allowances for Indians, a Report of the Expert Group of the Indian Council of Medical Research 2010. Hyderabad, India: National Institute of Nutrition; 2010.

32. Prakash J, Mehtani A, Sud A, Reddy BK. Is surgery always indicated in rachitic coronal knee deformities? Our experience in 198 knees. J Orthop Surg (Hong Kong). 2017; 25(1):2309499017693532. doi: 10.1177/2309499017693532. PMID: 28222650. 